\title{
Identification of fluorescent beads using a coded aperture snapshot spectral imager
}

\author{
Christy Fernandez Cull, ${ }^{1}$ Kerkil Choi, ${ }^{1}$ David J. Brady, ${ }^{1, *}$ and Tim Oliver ${ }^{2}$ \\ ${ }^{1}$ Fitzpatrick Institute for Photonics and Department of Electrical and Computer Engineering, \\ Duke University, 129 Hudson Hall, Durham, North Carolina 27708, USA \\ ${ }^{2}$ Department of Cell Biology, Duke University, 337 Nanaline Duke Building, Duke Box 3709, \\ Durham, North Carolina 27710, USA \\ *Corresponding author: dbrady@duke.edu
}

Received 1 October 2009; revised 12 January 2010; accepted 29 January 2010; posted 1 February 2010 (Doc. ID 118046); published 2 March 2010

\begin{abstract}
We apply a coded aperture snapshot spectral imager (CASSI) to fluorescence microscopy. CASSI records a two-dimensional (2D) spectrally filtered projection of a three-dimensional (3D) spectral data cube. We minimize a convex quadratic function with total variation (TV) constraints for data cube estimation from the 2D snapshot. We adapt the TV minimization algorithm for direct fluorescent bead identification from CASSI measurements by combining a priori knowledge of the spectra associated with each bead type. Our proposed method creates a 2D bead identity image. Simulated fluorescence CASSI measurements are used to evaluate the behavior of the algorithm. We also record real CASSI measurements of a ten bead type fluorescence scene and create a $2 \mathrm{D}$ bead identity map. A baseline image from filtered-array imaging system verifies CASSI's 2D bead identity map. ( 2010 Optical Society of America

OCIS codes: $\quad 100.3010,100.3190,110.0180,110.1758,110.4234,180.2520$.
\end{abstract}

\section{Introduction}

Fluorescence microscopy is a high contrast imaging tool where a light source is used to excite various parts of a sample labeled with fluorescent markers. With the emergence of new fluorescent markers for microscopy applications, one underlying challenge is to identify spectrally overlapping fluorescence emitters. Another challenge involves separating both weak and strong emitters from an autofluorescent background. Spectral imaging (SI) addresses these challenges by reconstructing a spectrum at every pixel location in an image. Most fluorescence microscopy applications associated with spectral imaging create a $2 \mathrm{D}$ spectral identity map for an end-user interested in target localization and characterization of a detector measurement. SI emerged in the field of remote sensing [1,2] and reconnaissance applications [3], and it has recently impacted biomedical imaging

0003-6935/10/100B59-12\$15.00/0

(C) 2010 Optical Society of America in pathology [4], gene mapping [5], and confocal fluorescence imaging of bacteria cells [6].

Traditionally, in SI a three-dimensional (3D) data cube (2D spatial, 1D spectral) is generated via scanning or filter-based approaches. Common scanning methods include tomographic, pushbroom, and whiskbroom imaging systems [7-9]. A major drawback of these systems is long acquisition times. In recent advances, spatial and spectral resolution from filter-based systems such as acousto-optic tunable filters (AOTFs) and liquid crystal tunable filters (LCTFs) have improved, with cost continuing to be a major drawback $[10,11]$. Current spectral imaging systems for fluorescence microscopy are scanning confocal systems that combine multiple excitation sources, filters, and a spectrograph for data cube estimation. Recent developments of snapshot spectral imagers address the challenges for both scanning and filter-based approaches [12-14].

We have developed various coded aperture snapshot spectral imagers (CASSI). Initially, we used a static binary-valued Hadamard mask for 
high-throughput analysis of weak and incoherent sources for spectrum recovery [15]. More recently, we used a static binary-valued, pseudorandom mask in CASSI for video rate spectral imaging of burning candles and for spectral analysis of microspheres used in fluorescence microscopy [16-18]. The mask then serves as both a high-throughput light collector and as a 3D spatial-spectral data cube encoder. CASSI overcomes the drawbacks associated with both scanning and filter-based systems since a data cube is acquired in a snapshot image at a low system cost. CASSI delineates from other snapshot spectral imagers [12-14] with an on-axis architecture, the use of direct encoding to balance trade-offs in spatial and spectral resolution and the use of constrained optimization for image estimation. A snapshot architecture makes CASSI well-suited for 3D data cube estimation of dynamic fluorescent scenes.

Our CASSI spectral engine uses a pixel coding strategy to record compressive measurements (2D) of a fluorescent scene (3D data cube). These measurements draw upon concepts from a compressive sensing (CS) paradigm where underdetermined measurements allow for highly accurate signal estimation $[19,20]$. Compressive measurements captured from $\overline{\mathrm{CA}} \overline{\mathrm{SSI}}$ systems use CS inversion with total variation (TV) minimization to reconstruct a $3 \mathrm{D}$ data cube from a single $2 \mathrm{D}$ image. In a previously reported experiment $[17,18,21]$, we adapted a two-step iterative shrinkage/thresholding (TwIST) algorithm for $3 \mathrm{D}$ data cube estimation. In this paper, we propose another adaptation to a CS TV minimization algorithm for the identification of fluorescent beads. We incorporate $a$ priori spectral data into the inversion process in order to further impose sparsity along the spectral domain. The imposed sparsity enables a more accurate reconstruction from fewer measurements. A three-step procedure is then applied to the sparse data cube estimate to create a $2 \mathrm{D}$ spectral feature map of fluorescent beads. Our approach is designed to address applications in fluorescence microscopy associated with target identification rather than spectral data cube estimation.
This paper is organized as follows. In Section2, we review a dual-disperser CASSI optical architecture and describe the effect of the aperture code on a source spectral density. Section 3 summarizes the TV minimization algorithm and explains the adaptation to further impose sparsity on the data cube using $a$ priori spectral information. The generation of a $2 \mathrm{D}$ bead identification map is described. We also demonstrate the performance of our algorithm via simulation in Section 3. Further, we present the CASSImicroscope interface in Section 4. Section 5 shows CASSI fluorescent bead identification maps and contains baseline images recorded with two multispectral imaging systems. We use these baseline images to verify CASSI bead identification maps.

\section{System Model}

The system model for a dual disperser snapshot spectral imager has already been developed [16]. We describe a variant on the dual disperser architecture previously discussed - the use of two direct-view double Amici prisms as dispersing elements; see Fig. 1. The double Amici prisms are of equal and opposite dispersion $( \pm \xi)$. This section provides a summary for the dual-disperser CASSI system model. An object can be described by a three-dimensional power spectral density, $f_{0}(x, y, \lambda)$, where $x$ and $y$ denote spatial location and $\lambda$ represents the wavelength. After the first group of optics in arm 1 (see Fig. 1 ), $f_{0}$ is mapped to

$$
f_{1}(x, y, \lambda)=\iint f_{0}\left(x^{\prime}, y^{\prime}, \lambda\right) h_{1}\left(x, x^{\prime}, y, y^{\prime}, \lambda\right) \mathrm{d} x^{\prime} \mathrm{d} y^{\prime}
$$

The kernel $h_{1}\left(x, x^{\prime}, y, y^{\prime}, \lambda\right)$ is modeled by a shift invariant impulse response that represents propagation through unity magnification imaging optics and a dispersive element. Diffraction effects in the CASSI architecture and optical aberrations are neglected in the expression for $h_{1}$ :

$$
h_{1}\left(x, x^{\prime}, y, y^{\prime}, \lambda\right)=\delta\left(x^{\prime}-\left[x+\xi_{1}\left(\lambda-\lambda_{C}\right)\right]\right) \delta\left(y^{\prime}-y\right) \text {. }
$$

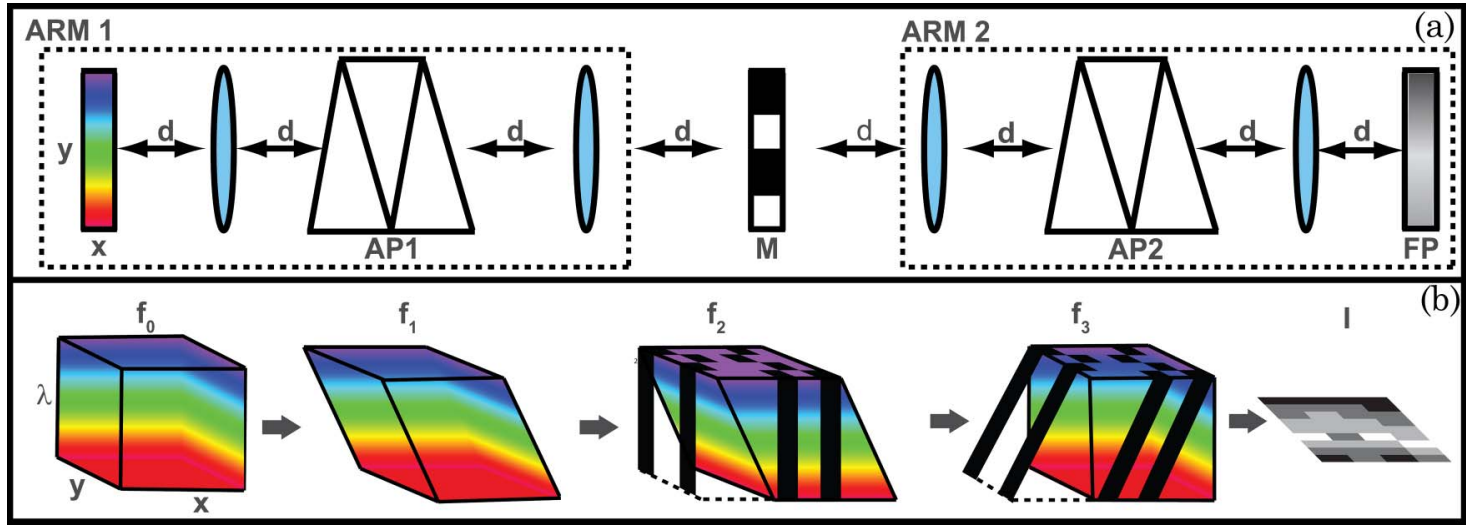

Fig. 1. (Color online) (a) Optical architecture for a dual disperser CASSI consisting of Amici prisms (AP1 and AP2), an aperture code ( $M$ ) and a focal plane (FP). (b) Power spectral density profile propagated through the system optical architecture. The effect of the aperture code on the power spectral density is illustrated. 
In Eq. (2), $\xi_{1}$ represents the net dispersion prescribed by the first double Amici prism and $\lambda_{C}$ is the system's center wavelength $(550 \mathrm{~nm})$. Dispersion by the Amici prism is expressed as $\mathrm{d} n / \mathrm{d} \lambda$. We assume linear dispersion in this system model for the prescribed spectral range of the instrument. We refer to the dispersion in arm 1 as a shearing operation along the $x$ dimension of the optical system. The power spectral density is neither magnified nor dispersed along the $y$ direction. By substituting Eq. (2) into Eq. (ㅁ) $f_{1}$ becomes

$$
\begin{aligned}
f_{1}(x, y, \lambda)= & \iint f_{0}\left(x^{\prime}, y^{\prime}, \lambda\right) \delta\left(x^{\prime}-\left[x+\xi_{1}\left(\lambda-\lambda_{C}\right)\right]\right) \\
& \times \delta\left(y^{\prime}-y\right) \mathrm{d} x^{\prime} \mathrm{d} y^{\prime} \\
= & f_{0}\left(x+\xi_{1}\left(\lambda-\lambda_{C}\right), y, \lambda\right) .
\end{aligned}
$$

After the prism, multiple images are formed at wavelength dependent locations. The sheared power spectral density is then modulated by an aperture code. A mask-modulated $f_{1}$ is expressed as

$$
f_{2}(x, y, \lambda)=t(x, y) f_{0}\left(x+\xi_{1}\left(\lambda-\lambda_{C}\right), y, \lambda\right),
$$

where $t(x, y)$ is the transmittance function of the aperture code. CASSI has a pseudorandom, binaryvalued transmittance function defined by

$$
t(x, y)=\sum_{i j}^{N} t_{i j} \operatorname{rect}\left(\frac{x-i \Delta_{T}}{\Delta_{T}}, \frac{y-j \Delta_{T}}{\Delta_{T}}\right),
$$

where $t_{i j}$ is the binary value ( 0 or 1 ) associated with either an opaque or a transmissive opening on the mask, $N$ refers to the total number of mask elements along each spatial dimension, and $\Delta_{T}$ refers to the mask element pitch. We refer to the mask modulation process as a punching operation, where spectral channels are blocked by opaque regions on the mask as a function of spatial location. The optics in arm 2 are meant to undo the dispersion effects from arm 1 (see Fig. 1). After the second Amici prism, $f_{2}$ becomes

$$
f_{3}(x, y, \lambda)=\iint f_{2}\left(x^{\prime \prime}, y^{\prime \prime}, \lambda\right) h_{2}\left(x, x^{\prime \prime}, y, y^{\prime \prime}, \lambda\right) \mathrm{d} x^{\prime \prime} \mathrm{d} y^{\prime \prime},
$$

$$
\begin{aligned}
f_{3}(x, y, \lambda)= & \iint t\left(x^{\prime \prime}, y^{\prime \prime}\right) f_{0}\left(x^{\prime \prime}\right. \\
& \left.+\xi_{1}\left(\lambda-\lambda_{C}\right), y^{\prime \prime}, \lambda\right) h_{2}\left(x, x^{\prime \prime}, y, y^{\prime \prime}, \lambda\right) \mathrm{d} x^{\prime \prime} \mathrm{d} y^{\prime \prime} .
\end{aligned}
$$

Recall that the Amici prism in arm 2 has equal and opposite dispersion to the Amici prism in arm 1 $\left(\xi_{2}=-\xi_{1}\right)$. In Eq. (8), $h_{2}$ is also modeled as the shift-invariant impulse response for the optics in arm 2:

$$
h_{2}\left(x, x^{\prime \prime}, y, y^{\prime \prime}\right)=\delta\left(x^{\prime \prime}-\left[x+\xi_{2}\left(\lambda-\lambda_{C}\right)\right]\right) \delta\left(y^{\prime \prime}-y\right) \text {. }
$$

Before the detector, $f_{3}$ is further simplified to

$$
f_{3}(x, y, \lambda)=f_{0}(x, y, \lambda) t\left(x-\xi_{1}\left(\lambda-\lambda_{C}\right), y\right) .
$$

We describe a spectrally dependent filter function as $H(x, y, \lambda)=t\left(x-\xi_{1}\left(\lambda-\lambda_{C}\right), y\right)$. The application of a $2 \mathrm{D}$ aperture code in a dispersive, intermediate image plane of CASSI creates a 3D spectrally shifted filter function that modulates a 3D power spectral density. Since the detector is insensitive to wavelength, the power spectral density at the detector is integrated along the wavelength dimension.

$$
g(x, y)=\int f_{0}(x, y, \lambda) H(x, y, \lambda) \mathrm{d} \lambda .
$$

We refer to this integration process at the detector as a smashing operation. Therefore, an intensity value at every pixel location is recorded at the detector. Since the measurements at the detector are spatially pixelated, we model the detector measurement as

$$
\begin{aligned}
g_{m n}= & \iiint t\left(x-\xi_{1}\left(\lambda-\lambda_{C}\right), y, \lambda\right) f_{0}(x, y, \lambda) \\
& \times p_{m n}(x, y) \mathrm{d} x \mathrm{~d} y \mathrm{~d} \lambda,
\end{aligned}
$$

where $p_{m n}$ represents the detector sampling function at the $(m, n)$ th location as described by

$$
p_{m n}(x, y)=\operatorname{rect}\left(\frac{x-m \Delta}{\Delta}, \frac{y-n \Delta}{\Delta}\right)
$$

and $\Delta$ represents the detector pixel pitch. Substituting Eqs. (13) and (6) into Eq. (12), we obtain

$$
\begin{aligned}
g_{m n}= & \sum_{i j} t_{i j} \iiint \operatorname{rect}\left(\frac{x-\xi_{1}\left(\lambda-\lambda_{C}\right)-i \Delta_{T}}{\Delta_{T}}, \frac{y-j \Delta_{T}}{\Delta_{T}}\right) \\
& \times \operatorname{rect}\left(\frac{x-m \Delta}{\Delta}, \frac{y-n \Delta}{\Delta}\right) f_{0}(x, y, \lambda) \mathrm{d} x \mathrm{~d} y \mathrm{~d} \lambda .
\end{aligned}
$$

Next, we assume that the mask element pitch $\left(\Delta_{T}\right)$ and the detector pixel pitch $(\Delta)$ are equal $\left(\Delta_{T}=\Delta\right)$. Ideally, we want to establish a relationship between the mask and detector sampling functions to simplify the expression in Eq. (14). To do this, we assume that $k=\xi_{1}\left(\lambda-\lambda_{C}\right) / \Delta$. After substituting $k$ into Eq. (14), we have

$$
\begin{aligned}
g_{m n}= & \sum_{i j} t_{i j} \iiint \operatorname{rect}\left[\frac{x-(k-i) \Delta}{\Delta}, \frac{y-j \Delta}{\Delta}\right] \\
& \times \operatorname{rect}\left[\frac{x-m \Delta}{\Delta}, \frac{y-n \Delta}{\Delta}\right] f_{0}(x, y, \lambda) \mathrm{d} x \mathrm{~d} y \mathrm{~d} \lambda .
\end{aligned}
$$

The sampling functions for the aperture code and the detector overlap when $m=k-i$ and $j=n$. In order to create a discrete representation of $f_{0}$, we substitute 
$x^{\prime}=x-m \Delta, \quad y^{\prime}=y-n \Delta, \quad$ and $\quad \lambda^{\prime}=\lambda-k \Delta \quad$ into Eq. (15), which yields

$$
\begin{aligned}
g_{m n}= & \sum_{i j} t_{i j} \delta_{i, k-m} \delta_{j, n} \iiint f_{0}\left(x^{\prime}+m \Delta, y^{\prime}+n \Delta, \lambda^{\prime}+k \Delta\right) \\
& \times \operatorname{rect}\left[\frac{x^{\prime}}{\Delta}, \frac{y^{\prime}}{\Delta}\right] \mathrm{d} x^{\prime} \mathrm{d} y^{\prime} \mathrm{d} \lambda^{\prime},
\end{aligned}
$$

where $\Delta$ again represents the detector pixel pitch. A discrete representation $(f)$ for the $3 \mathrm{D}$ object distribution $\left(f_{0}\right)$ is modeled as

$$
\begin{aligned}
f_{m, n, k}= & \iiint f_{0}\left(x^{\prime}+m \Delta, y^{\prime}+n \Delta, \lambda^{\prime}+k \Delta\right) \\
& \times \operatorname{rect}\left[\frac{x^{\prime}}{\Delta}, \frac{y^{\prime}}{\Delta}\right] \delta\left(\lambda^{\prime}+k \Delta\right) \mathrm{d} x^{\prime} \mathrm{d} y^{\prime} \mathrm{d} \lambda^{\prime} .
\end{aligned}
$$

We further simplify the detector measurements using the following equations:

$$
\begin{gathered}
g_{m n}=\sum_{i j k} t_{i, j} f_{m, n, k} \delta_{i, k-m} \delta_{j, n}, \\
g_{m n}=\sum_{k} t_{k-m, n} f_{m, n, k} .
\end{gathered}
$$

This discrete model for CASSI measurements describes the effect of the aperture code on an input power spectral density. Since we have defined our aperture code function $(t)$ as a 3D filter function $(H)$ in Eq. (11), our CASSI measurements may be algebraically written as

$$
g=H f .
$$

Note that diffraction effects located at the sample plane of the microscope are relayed to the image plane of CASSI. These diffraction effects are not incorporated into the CASSI system model. Diffracted objects at the sample plane appear defocused at the CASSI image plane. A defocused object results in a larger spectrally encoded object detected by CASSI. An enlarged object does not impact the spectral feature identification algorithm detailed in this paper. CASSI measures a smaller number of voxels required to represent the signal $f$. A CS inversion scheme is used to solve this underdetermined problem using TV minimization. Postprocessing steps to generate a spectral feature map from a $3 \mathrm{D}$ data cube estimate are discussed in the next section.

\section{Reconstruction Procedure and Simulation Results}

\section{A. 3D Data Cube Estimation}

We have adapted the TwIST TV minimization algorithm [21] for data cube estimation from a single 2D image [18]. The data-cube estimate provides a spatial $(x, y)$ and spectral $(\lambda)$ representation of an object. Using TwIST [21], we minimize a convex objective function defined by

$$
f^{*}=\arg \min _{f}\|g-H f\|_{2}^{2}+\tau \Phi_{\mathrm{TV}}(f),
$$

where $H$ is our projection matrix, $g$ is the CASSI measurement, and $\tau$ is the regularizing parameter. In the nonlinear convex optimization algorithm we use TV minimization to find a sparse gradient solution to Eq. (21). $\Phi_{\mathrm{TV}}$ is defined by

$$
\Phi_{\mathrm{TV}}(f)=\sum_{k} \sum_{i, j} \sqrt{\left(f_{i+1, j, k}-f_{i, j, k}\right)^{2}+\left(f_{i, j+1, k}-f_{i, j, k}\right)^{2}} .
$$

While previous data cube reconstructions were reasonable, 2D TV minimization does not impose sparsity along the spectral domain. As a result, a larger number of measurements is required to reconstruct a more accurate data cube from a $2 \mathrm{D}$ snapshot. This challenge provides the motivation to further impose sparsity on the data cube along the spectral domain. This is addressed by incorporating prior knowledge into the inversion process as described in subsequent sections.

\section{B. Direct Spectral Feature Identification Algorithm}

In fluorescence microscopy, fluorochrome spectral profiles in a sample are known a priori. We use this a priori knowledge to enable more accurate data cube estimation and $2 \mathrm{D}$ spectral feature identification. This section aims to describe a three-step procedure to produce a $2 \mathrm{D}$ spectral feature map of a fluorescence scene where a unique value at every pixel location only corresponds to one vector in the spectral database, $W$ (see Fig. 2).

First, we adapt a $2 \overline{\mathrm{D}}$ TV minimization algorithm for data cube estimation by incorporating spectral priors into the inversion scheme. To do this, we transform an object data cube, $f(i, j, k)$, into another data cube, $\alpha\left(m_{1}, m_{2}, n\right)$. The location along the $n$ axis represents color identification for a spectral vector, and the value of $\alpha\left(m_{1}, m_{2}, n\right)$ represents the brightness. Figure 2 provides an example for a spectral database, $W$. $W$ is represented as $W=\left\{s_{n}\right\}_{n=1}^{T}$, where $T$ corresponds to the total number of spectral vectors in $W$. Each spectral vector, $s_{n}$, represents a column vector in $\Re^{L}$. Our goal for inversion is not to reconstruct the $f$ data cube, but rather our hope is to reconstruct a more accurate data cube, $\alpha^{*}$ [see Eq. (26)]. The data cube estimate, $\alpha^{*}$, should tend to be more accurate since it is a sparser data cube. More accurate estimation is facilitated when fewer nonzero values can be used to represent an object data cube. CS theory indicates that the object sparsity in a chosen basis is a crucial factor for reconstruction accuracy $[19,20]$. In other words, a sparser representation of the object can provide a more accurate estimate when the total number of measurements is fixed. Note that the transformation of the $f$ data cube into the $\alpha$ data cube generally reduces the sparsity in $f$ on the TV basis. We algebraically express the transformation of $f$ as 


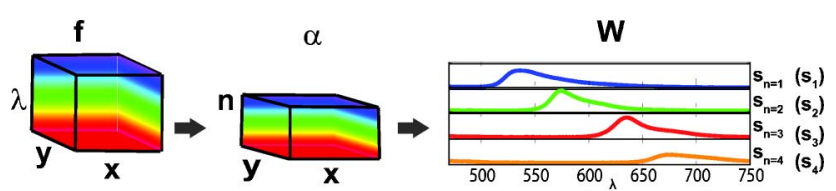

(a)

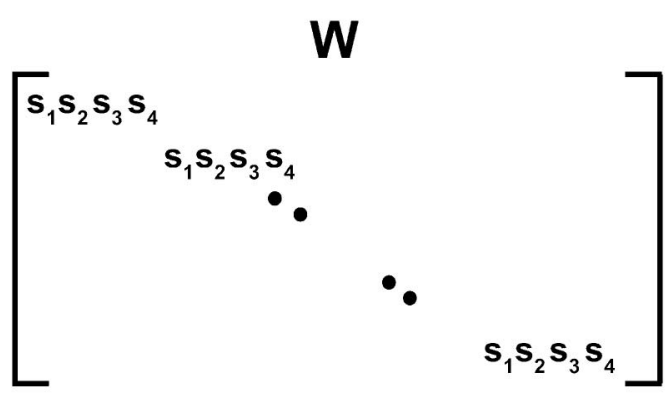

(b)

Fig. 2. (Color online) (a) Object data cube $(f)$ transformation into a sparse data cube $(\alpha)$ using spectral priors $(W)$. A spectrum recorded at a single pixel location in $f$ corresponds to a single pixel value/bead identity in $\alpha$. (b) Matrix representation of the spectral data base, $W$.

$$
f=W \alpha,
$$

where $W$ represents a block diagonal matrix of spectral column vectors [see Fig. 2(b)] and $\alpha$ represents a sparse data cube. Note that once $\alpha$ is estimated, $f$ is retrieved via matrix multiplication.

Then, we modify the 2D TV minimization algorithm in Eq. (21) by incorporating the transformation of $f$ into forward and transpose models. We define CASSI's new forward model as

$$
g=H W \alpha,
$$

where the system's transformation matrix $(H)$ is characterized by a summation operator $(V)$ and a calibration cube $(C) . V$ is a matrix operation representing the smashing operation at the detector, and $C$ is a matrix describing a 2D aperture code shifted as a function of spectral channel $(k)$. Actual implementation of the forward model consists of replicating each normalized spectral vector within the set $\left\{s_{n}\right\}$ at locations spanning the spatial extent of the detector measurement $(g)$, thereby creating a cube for each spectral vector, $s_{n}(i, j, k)$. Recall that the length of $k$ corresponds to the length of $s_{n}(L)$. An inner product is taken between each spectral vector cube and the corresponding $n$ channel in $\alpha . \alpha_{n}$ is equivalent to $\alpha\left(m_{1}, m_{2}, k\right)$, where the 2D slice for the $n$ channel estimate is replicated $L$ times so that an inner product between a spectral vector cube and an $\alpha_{n}$ cube can be computed. Results produced by the inner products are then added. Afterwards, the result is element-wise multiplied by $C$, and a sum is taken along the $k$ dimension to provide a new estimate for the detector image, $g$. The transpose model for CASSI is given by

$$
(H W)^{T}=W^{T} C^{T} V^{T} .
$$

Further, the spectral database $(W)$, detector estimate $(g)$, and calibration cube $(C)$ are used as inputs to the transpose model. A $g$ data cube is created by replicating the measurement matrix by the number of spectral channels $(k)$ that CASSI measures. The $g$ data cube is then multiplied by $C$. Then, we compute the inner product between each spectral signature cube, $s_{n}(i, j, k)$, and the mask-modulated $g$ cube in order to provide a channel estimate $(n)$. Lastly, a sum is taken along the $k$ domain to generate a quasi estimate for each channel $(n)$ in $\alpha$. This new estimate is in the same domain as $\alpha^{*}\left(m_{1}, m_{2}, n\right)$. The forward and transpose models are implemented at every iteration in the TV minimization algorithm as described by

$$
\alpha^{*}=\arg \min _{\alpha}\|g-H W \alpha\|_{2}^{2}+\tau \Phi_{\mathrm{TV}}(\alpha),
$$

where the transformation of $f$ is adapted.

The second step for generating a $2 \mathrm{D}$ spectral feature map is to create a maximum value cube, $\beta$, from the algorithm estimate, $\alpha^{*}$. Recall that $\alpha^{*}$ corresponds to a sparse data cube representation of $f$ via a spectral basis decomposition $(W)$, which is calculated via an inner product between each spectral signature, $s_{n}$, with the measured data, $g$. A single value is obtained from each inner product at each pixel location. This value corresponds to the similarity between each spectral signature with the measured data. While physically the $\left(m_{1}, m_{2}\right)$ pixel location should only correspond to a single $n$ value, the algorithm estimate $\left(\alpha^{*}\right)$ may yield residual values in neighboring channels. For significantly broad and overlapping spectral vectors in $W$, the residual values near channel $n$ may be large. Once an $\alpha^{*}$ estimate is produced, we find the maximum values along the $n$ axis at each $\left(m_{1}, m_{2}\right)$ location. We create a maximum value cube, $\beta$, using

$$
\beta\left(m_{1}, m_{2}, n\right)=\max _{n} \alpha^{*}\left(m_{1}, m_{2}, n\right) .
$$

In $\beta$ only one nonzero value is found along the $n$ axis for every $\left(m_{1}, m_{2}\right)$ location. Recall that each position along $n$ corresponds to a color identification for a spectral vector, and each $\beta$ value represents the brightness associated with each $\left(m_{1}, m_{2}\right)$ location. We can create two different spectral feature maps, $\gamma^{*}$ from $\beta$.

Our last step involves producing two different spectral feature maps, $\gamma_{1}^{*}$ and $\gamma_{2}^{*}$. Our objective is to create a $2 \mathrm{D}$ color-coded map that identifies each bead type for an end-user. Thus, we create a brightness and color preserving feature map $\left(\gamma_{1}^{*}\right)$ and a color-only feature map $\left(\gamma_{2}^{*}\right)$. For the brightness preserving feature map [see Fig. 7(b)], we use the hue, saturation, and value (HSV) model. The maximum brightness value $\beta$ is the input for value, and the position along $n$ corresponds to the input for hue in the HSV model. We set saturation for each $\left(m_{1}, m_{2}\right)$ 
position to one. An HSV model is generated for each $n$ slice in $\beta$. Each HSV map is converted into an RGB equivalent where brightness and color information are preserved. Thus, we map $\beta\left(m_{1}, m_{2}, n\right)$ to $\gamma_{1}\left(m_{1}, m_{2}, n\right)$ using an HSV map and an HSV to RGB mapping. Note that three values are associated with each $\left(m_{1}, m_{2}\right)$ location for the $n$th channel RGB map. To create a $2 \mathrm{D}$ spectral feature map, $\gamma_{1}^{*}$, we sum each RGB map created for each $n$ channel using

$$
\gamma_{1}^{*}\left(m_{1}, m_{2}\right)=\sum_{n} \gamma_{1}\left(m_{1}, m_{2}, n\right) .
$$

We also create a second spectral feature map $\left(\gamma_{2}^{*}\right)$ based on color. Values in $\beta$, along each $n$ channel, are normalized and multiplied by the $n$ value assigned to a single spectral vector. We transform $\beta\left(m_{1}, m_{2}, n\right)$ into $\gamma_{2}\left(m_{1}, m_{2}, n\right)$, where each nonzero value in $\gamma_{2}$ corresponds solely to color:

$$
\gamma_{2}\left(m_{1}, m_{2}, n\right)=n\left[\frac{\beta\left(m_{1}, m_{2}, n\right)}{\max _{n} \beta\left(m_{1}, m_{2}, n\right)}\right] .
$$

We create a pseudocolor map by assigning $n$ vectors in a linear gray-scale color map to unique RGB values [see Fig. 7(c)]. The 2D spectral feature map, $\gamma_{2}^{*}$, is created by performing a summation along the $n$ axis in $\gamma_{2}$ using

$$
\gamma_{2}^{*}\left(m_{1}, m_{2}\right)=\sum_{n} \gamma_{2}\left(m_{1}, m_{2}, n\right)
$$

By doing this, the intensity across a single bead is assigned a constant value and any intensity variation across the bead is not preserved. Lastly, we display the 2D image using the prescribed color map.

In summary, this section described a three-step approach to create two, 2D spectral feature maps $\left(\gamma_{1}^{*}\right.$ and $\left.\gamma_{2}^{*}\right)$ of a fluorescence scene. The first step involved a modification to a $2 \mathrm{D}$ TV minimization algorithm by incorporating spectral priors into the inversion scheme. As a result, $f(i, j, k)$ was transformed into a sparser data cube, $\alpha\left(m_{1}, m_{2}, n\right)$. Second, a maximum value cube was created from the algorithm estimate. The last step in the procedure involved creating two, 2D spectral feature maps from the maximum value cube. Simulations based on the proposed algorithm are discussed in the next section.

\section{Simulation Results}

A TwIST 2D TV minimization algorithm incorporating spectral priors is tested on simulated data to understand the behavior of the algorithm. We simulate a $3 \mathrm{D} \alpha$ data cube by creating a $64 \times 64$ pixel scene of four squares containing spectra from the database in Fig. 3(b). The spectral signatures are downsampled spectra corresponding to calibration standards used in fluorescence microscopy. Simulated detector measurements are produced using the aforementioned forward model. We simulate the calibration cube as a shifted $64 \times 64$ pixel aperture code. A spectral shift of $10 \mathrm{~nm}$ corresponds to a single pixel shift of the aperture code. A cropped $64 \times 64$ pixel replica of the CASSI aperture code is used in simulation. Also, our simulated system model measures 22 spectral channels spanning the spectral range between $490 \mathrm{~nm}$ and $700 \mathrm{~nm}$ with $10 \mathrm{~nm}$ spectral resolution. We evaluate the reconstructed estimate $\left(\alpha^{*}\right)$ using mean squared error (MSE).

In simulation, we analyze estimation efficacy for two different square sizes. Noise is not added to CASSI measurements for this analysis. We compare $10 \times$ 10 and $15 \times 15$ pixel squares to quantify how bead size might impact reconstruction accuracy. Regularization parameters $(\tau)$ were chosen by trial and error to visually produce the best reconstruction results. Number of iterations $(p)$ is chosen such that the relative difference in the objective function is nominal. The simulated $3 \mathrm{D} \alpha$ data cube is compared to the estimated 3D $\alpha^{*}$ data cube using MSE. The calculated MSE for the $10 \times 10$ pixel squares is 0.1167 and for the $15 \times 15$ pixel squares is 0.0415 . Note that we obtain a smaller MSE for the estimation of larger squares embedded in a $64 \times 64$ pixel scene as depicted in Fig. 4 . The analysis of square size provides the motivation to test large beads in the fluorescent microsphere experiment detailed in Section 5.

Reconstruction accuracy measured using MSE is a function of object sparsity in the chosen basis (i.e., TV in our study). In simulation, we can analyze estimates by measuring object sparsity in the TV basis. The number of nonzero gradients, or level of object sparsity, in the TV basis is calculated for two different square size objects. A nonzero gradient at the $(i, j)$ th pixel in the $k$ th spectral slice, $\left|\nabla f_{i, j, k}\right|$, is defined as

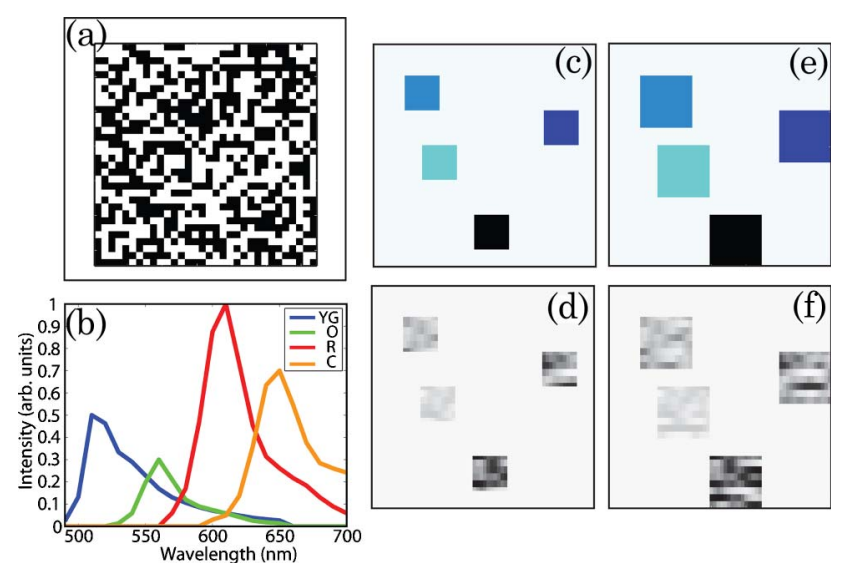

Fig. 3. (Color online) (a) Simulated $64 \times 64$ pixel aperture code. (b) Downsampled fluorescence spectra of a 0.3 intensity-valued yellow green $(\mathrm{YG} / n=1), 0.5$ intensity-valued orange $(\mathrm{O} / n=2)$, 1.0 intensity-valued red $(\mathrm{R} / n=3)$ and 0.7 intensity-valued crimson $(\mathrm{C} / n=4)$ beads. (c) Simulated $10 \times 10$ pixel fluorescent squares in a $64 \times 64$ pixel image with the corresponding (d) simulated detector image. (e) Input image for $15 \times 15$ pixel spectrally different fluorescent squares in a $64 \times 64$ pixel image with the corresponding (f) simulated detector image. 


$$
\left|\nabla f_{i, j, k}\right|=\sqrt{\left(f_{i+1, j, k}-f_{i, j, k}\right)^{2}+\left(f_{i, j+1, k}-f_{i, j, k}\right)^{2}} .
$$

Note that larger squares produce more nonzero detector measurements $\left(g_{m . n}>0\right)$ and more nonzero gradients. For the $10 \times 10$ pixel squares in Fig. 3(c), we calculated 174 nonzero gradients in the TV basis and 396 nonzero values for the detector measurement, $g$. Similarly, we measured 252 nonzero gradients in the TV basis for the $15 \times 15$ pixel squares and compared this number to 896 nonzero detector measurements. A ratio of nonzero detector measurements to object nonzero gradients in the TV basis suggests a measure for reconstruction accuracy. In $\mathrm{CS}$, the number of measurements required for adequate signal estimation is $M \approx O[K \log (N / K)]$, where $K$ represents the sparsity of the signal in some basis and $N$ represents the original size of the signal. Larger squares provide a larger number of measurements and a higher measurement to object gradient ratio, which is an indication for improved CS signal estimation. Figure 4(b) shows a reconstruction estimate for $10 \times 10$ pixel squares. The dotted line in the $\alpha^{*}(n=4)$ estimate represents reconstruction error. Contained within the dotted line is a residual square from the $n=3$ channel estimate. We show that an increase in the number of nonzero detector measurements improves reconstruction accuracy in Fig. 4(d). The ratio of total detector measurements to object sparsity in the TV basis directly impacts TV minimization reconstructions.

Also, we compare previous work in 3D data cube estimation $\left(f^{*}\right)$ to TV minimization reconstruction with spectral priors $\left(\alpha^{*}\right)$. First, we calculate the MSE between our simulated $f$ data cube $\left(f_{T}\right)$ and the $f^{*}$ data cube estimate. Next, we transform our TV minimization estimate $\left(\alpha^{*}\right)$ into a $f_{\alpha}^{*}$ data cube estimate using Eq. (23), and we calculate MSE between $f_{T}$ and $f_{\alpha}^{*}$. Without noise, the MSE for the $f_{\alpha}^{*}$ data cube is 0.1392. This MSE is an order of magnitude better

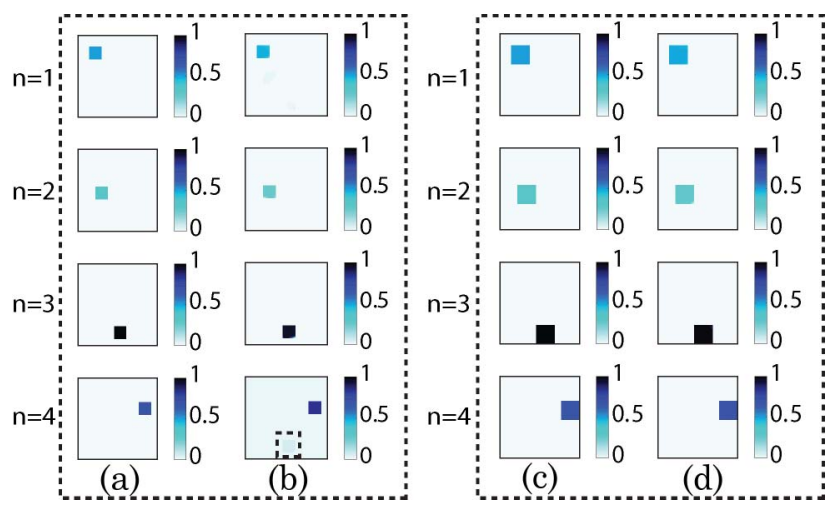

Fig. 4. (Color online) Simulated $\alpha$ and reconstructed $\alpha^{*}$ data cube, where each $n$ channel relates to a single spectral vector in $W$. (YG, $n=1 ; \mathrm{O}, n=2 ; \mathrm{R}, n=3 ; \mathrm{C}, n=4)$ (a) "true" $\alpha\left(m_{1}, m_{2}\right)$ as a function of $n$ for $10 \times 10$ pixel squares (b) estimated $\alpha^{*}\left(m_{1}, m_{2}\right)$ as a function of $n$ for $10 \times 10$ pixel squares. The dotted line in the $n=4$ slice represents a residual artifact from the $n=3$ slice. (c) "true" $\alpha\left(m_{1}, m_{2}\right)$ as a function of $n$ for $15 \times 15$ pixel squares (d) estimated $\alpha^{*}\left(m_{1}, m_{2}\right)$ as a function of $n$ for $15 \times 15$ pixel squares. than the MSE obtained with the $f^{*}$ data cube, which is equal to 0.0331 .

Further, we analyze the impact of noise on TV minimization reconstruction. Poisson noise is added to CASSI measurements. Poisson noise is generated with signal-to-noise ratio (SNR) defined by

$$
\mathrm{SNR} \stackrel{\text { def }}{=} 10 \log _{10}\left\{\frac{\sum_{m} \sum_{n} g_{m, n}^{2}}{\sum_{m} \sum_{n}\left(g_{m, n}^{p}-g_{m, n}\right)^{2}}\right\}
$$

where

$$
g_{m, n}^{p} \sim \frac{1}{\eta} \operatorname{Poisson}\left\{\eta g_{m, n}\right\} .
$$

The constant value $\eta$ is chosen based on a desired measurement SNR. We add Poisson noise to CASSI measurements because our experimental system is shot-noise limited. We compare the impact of noise on reconstruction efficacy for two different data cube estimation methods (i.e., $f^{*}$ and $f_{\alpha}^{*}$ estimation). In Fig. 5, we analyze 18 different measurement SNR noise levels and consider three different values for $\tau$. We observed that multiple reconstruction MSEs made at each noise level under the same algorithm parameters are consistent. The $f_{\alpha}^{*}$ data cube MSE values are still an order of magnitude better then MSE values obtained with $f^{*}$ reconstructions. Reconstruction MSE converges for both data cube estimation methods around a $40 \mathrm{~dB}$ measurement SNR. Also, the computational cost for estimating $f^{*}$ is twice as expensive as the estimation of $f_{\alpha}^{*}$. This factor for computational cost varies with image size. These simulation results show that the estimation of $\alpha^{*}$ produces a more accurate data cube estimate of a simulated $f$ data cube compared to $f^{*}$ data cube estimation.

In Section 3 , we provided motivations for the spectral feature identification algorithm. Two methods to create a 2D spectral feature map were described. We described our implementation for forward and transpose models used to estimate $\alpha^{*}$. Algorithm simulations describe the performance of the proposed technique. Simulation results reveal the importance of object size for accurate signal estimation and show the impact of Poisson noise on reconstruction accuracy. We did not explore spectral signature limitations in this section. In Section 5, we present reconstructions from real CASSI measurements containing beads with significantly overlapping spectra.

\section{System Design}

A custom-designed, $f / 11$ CASSI is coupled to the output port of a Zeiss AxioObserver A1 inverted microscope as shown in Fig. 6. A Xenon (XBO) arc lamp is used as an excitation source for fluorescence scene analysis. Light from the lamp is directed towards a filter cube located below the objective turret. The filter cube contains an excitation filter, dichroic filter, and an emission filter. The spectral range for the 

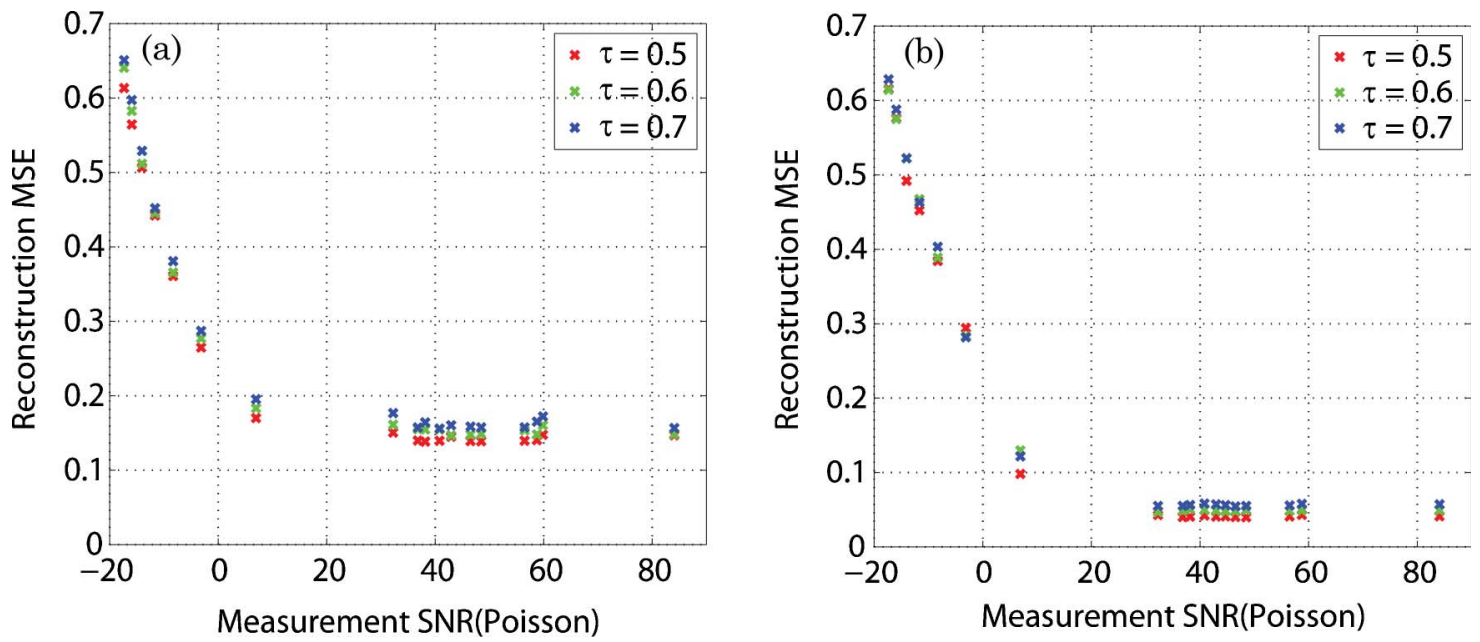

Fig. 5. (Color online) Plot of reconstruction MSE from CASSI measurements corrupted by Poisson noise. Reconstruction efficacy is compared between (a) direct $f^{*}$ data cube estimation and (b) $f_{\alpha}^{*}$ data cube estimation (see Subsection $\underline{3 . C}$ for the definition of $f_{\alpha}^{*}$ ).

excitation light is between $377 \mathrm{~nm}$ and $396 \mathrm{~nm}$, and the emission filter spectral bandwidth ranges between $420 \mathrm{~nm}$ and $900 \mathrm{~nm}$. Fluorescence signals from the sample are collected by a microscope objective and redirected towards three intermediate image planes of the microscope. The three intermediate image planes are located at two different exit ports and at the eyepiece. CASSI is coupled to an exit port on the inverted microscope [see Figs. 6(c) and 6(d)]. Two detectors are interfaced with CASSI using a flip mirror. The first detector is a cooled, scientific camera used for imaging samples that require longer exposure times. The detector operates at a resolution of $1200 \times 1600$ pixels (16-bit dynamic range) with a pixel pitch of $7.4 \mu \mathrm{m}$. A high frame rate camera is used for dynamic scene analysis. It operates at 15 FPS at full resolution $(2048 \times 2048)$ with a pixel pitch of $7.4 \mu \mathrm{m}$. The optical architecture and ray-traced model for CASSI is described in Figs. 1(a) and 6(b). CASSI uses two direct-view double Amici prisms made

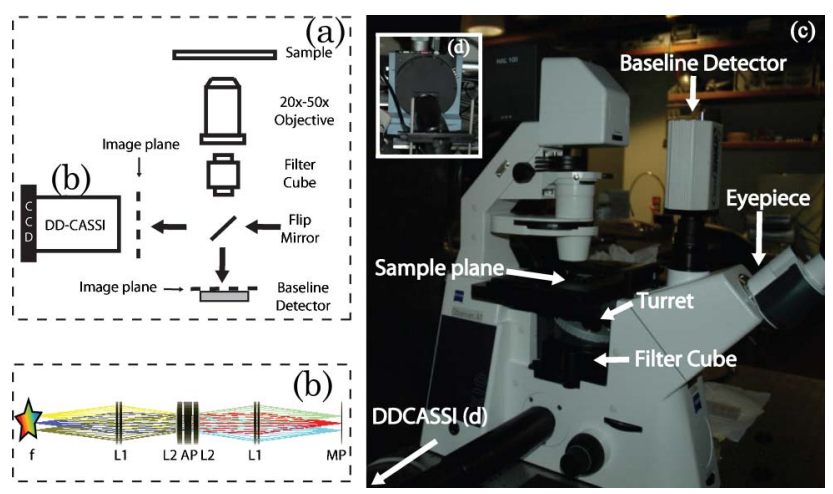

Fig. 6. (Color online) (a) Optical architecture for a CASSI interface to an inverted microscope. (b) Realization of Fig. 1(a) is in this ray-traced drawing for the first half of CASSI where $(f)$ is the object $(L 1)$ and (L2) are imaging and collimating lenses, (AP) is a directview double Amici prism and (MP) is the mask plane where the aperture code resides. (c) Layout of a Zeiss AxioObserver microscope with CASSI coupled to an exit port. (d) Back end of CASSI. from BK7 and SF6 materials. Light from the object is dispersed and imaged onto the aperture code located at the mask plane (MP) of the optical design. At the mask plane, the dispersion for the spectral range between $450 \mathrm{~nm}$ and $750 \mathrm{~nm}$ is about $499 \mu \mathrm{m}$. A mask element is mapped to two pixels at the detector plane $(14.8 \mu \mathrm{m})$. With these specifications, CASSI measures about 31 spectral channels within the spectral band of $450 \mathrm{~nm}-750 \mathrm{~nm}$ with a spectral resolution of about $10 \mathrm{~nm}$. Recall that the second half of the optical design is a mirror image of the first half [see Fig. 6(b)] consisting of a double Amici prism with equal and opposite dispersion. The resolution spot sizes across the field of view (FOV) of CASSI vary as a function of spatial position and wavelength. At the edges of the spectral range and at the edges of CASSI's FOV, the average spot size is about $16 \mu \mathrm{m}$. Typically, the spatial resolution of coded aperture systems is limited by the mask element size $(14.8 \mu \mathrm{m})$. Along the edges of the system's FOV and at the two edges of the the spectral range, the optical resolution of the system becomes the limiting factor and image quality is degraded. Some of the fluorescent beads analyzed in Section 5 are affected by the limitations of CASSI's optical resolution.

\section{Experimental Results}

\section{A. Calibration Procedure and Algorithm Implementation}

The CASSI model described in Section 2 is based on a variety of assumptions. We assume a $1: 1$ correspondence between the mask element pitch and the detector pixel pitch. In practice, not all wavelengths spanned by an input power spectral density correspond to single pixel shifts at the detector. Also, a unity magnification impulse response assumed for both arms $\left(h_{1}\right.$ and $\left.h_{2}\right)$ in the system model does not account for a spatially varying blur, a change in magnification, or aberrations in the system. A calibration cube for CASSI is taken before a fluorescence scene is analyzed. Ideally, a calibration cube would consist of 
translating a monochromatically illuminated fiber across the system's FOV at wavelengths spanning the spectral range of the instrument. However, this calibration process is photon-limited and time consuming. We acquire a calibration cube via full-field monochromatic illumination of CASSI. Each spectral slice in the cube represents a spectral impulse response recorded every $5 \mathrm{~nm}$ for the spectral range between $450 \mathrm{~nm}$ and $750 \mathrm{~nm}$. Unlike the monochromatic source analysis explored in Section 2, multispectral objects measured by CASSI can be described as a superposition of shifted mask patterns that span the object's spectrum. The calibration cube considers the influence of a spectrally shifted aperture code on a multispectral object.

For fluorescent target analysis, we use the TwIST TV minimization algorithm with the modification discussed in Section 3. Inputs to the algorithm include a CASSI measürement, a calibration cube, a regularization constant, and a spectral database. A region in the image is cropped in order to apply local processing. This is possible because the detector measurements are well registered. Although full FOV processing is possible, local processing provides a lower computational cost. For low contrast signals, a cropped region in an $\alpha^{*}$ estimate is renormalized to locally increase the contrast of a weak signal. This procedure is similar to applying a local digital gain to an image. By renormalizing the cropped region, all brightness information from the sparse data cube estimate is removed. These inputs and techniques are used for more accurate data cube estimation and for $2 \mathrm{D}$ spectral feature mapping of a ten bead type fluorescence scene.

There are multiple noise contributions that affect CASSI images recorded with a cooled, scientific camera. Two most common noise contributions are photon noise and detector noise. With a well depth of $2^{16}$, a readout noise of $\left(7.9 e^{-}\right)^{2}$, and a dark current of $\left(3 e^{-}\right)^{2}$, the detection system is shot-noise limited. The effect of dark current noise is minimized with additional cooling of the detector to $-20^{\circ} \mathrm{C}$. We subtract a background signal with mean 124 electrons from each data set to minimize readout noise and dark current noise contributions. Readout noise and dark current noise have nominal effects. Also, we use longer exposure times to maximize our SNR. While concerns for the impact of readout noise and dark current are emphasized in fluorescence microscopy of biological samples, in this study, the sample fluorescence emission is photon abundant. Simulations in Section 3 describe the impact of Poisson noise on reconstruction efficacy for a shot-noise limited measurement. If a photon-limited case was tested, readout noise would become significant. In this case, TV-minimization reconstructions would be more affected by noise contributions.

\section{B. Fluorescence Microscopy with CASSI}

We prepare our sample slide using Invitrogen Molecular Probes fluosphere solutions. We chose ten spec- trally different fluorescent microsphere solutions. Each fluosphere solution contained $15 \mu \mathrm{m}$ polystyrene microspheres/beads suspended in $10 \mathrm{~mL}$ of $0.15 \mathrm{M} \mathrm{NaCl}$ with $0.05 \%$ Tween 20 and $0.02 \%$ thimerosal. First, the ten different bead solutions were mixed in a separate container. Then, a mixed solution of fluorescent beads was mounted onto a slide using a FluorSave mounting medium from Calbiochem. We analyzed the microscope slide at the "best focus" FOV in the image plane of the microscope objective. We define a "best focus" FOV as a plane empirically found when a majority of the beads come to focus. Invitrogen's absorption and emission maximum wavelengths are described in Table 1 . Unlike current multispectral systems used in microscopy, CASSI fluorescence bead experiments employ a single, UV excitation source to excite all ten bead types mentioned in Table 1. One excitation source removes the need for multiple emission filters or movable parts typically needed in confocal spectral microscopy setups. Since we apply a single excitation scheme, we record baseline UV excited emission spectra for each microsphere type using an Ocean Optics (OO) slit spectrometer (see Table 1). The recorded $\mathrm{OO}$ spectral emissions [see Fig. $7 \overline{(\mathrm{e})}$ ] are inserted into the spectral database $(W)$ for sparse data cube estimation and bead identification. Note that CASSI can be adapted for multisource narrowband excitation. A single wide-band UV source merely simplified the implementation for the ten bead type sample under test.

In our experimental setup, we interface CASSI to an upright microscope for fluorescence scene analysis of calibration standards and apply a 2D TV minimization algorithm using spectral priors to the measured data. Sample information is relayed into an intermediate image plane of the microscope using a Zeiss LD Epiplan 50×, $0.5 \mathrm{NA}$ air objective. The fluorescent signal at the sample plane is relayed to an intermediate image plane located at an output port of the microscope. Recall that a "best focus" FOV is chosen with the microscope objective. In Figs. 7(c) and 7(d), some beads appear larger than the rest of the beads contained within a selected FOV. The Y4 bead size in Fig. 7(a) is caused by an

Table 1. Fluorescent Microspheres

\begin{tabular}{llcccc}
\hline & & & $\begin{array}{c}\text { Invitrogen (I) } \\
\text { Abs/Em }(\mathrm{nm})\end{array}$ & $\begin{array}{c}\text { OO Ex/Em } \\
(\mathrm{nm})\end{array}$ & $\begin{array}{c}{[\mathrm{OO}-\mathrm{I}]} \\
(\mathrm{Em})\end{array}$ \\
\hline 1 & Blue-green & $\mathrm{BG}$ & $430 / 465$ & $390 / 485$ & +20 \\
2 & Green & $\mathrm{G}$ & $450 / 480$ & $390 / 518$ & +38 \\
3 & Yellow-green & YG & $505 / 515$ & $390 / 537$ & +22 \\
4 & Yellow & $\mathrm{Y}$ & $515 / 534$ & $390 / 606$ & +72 \\
5 & Orange & $\mathrm{O}$ & $540 / 560$ & $390 / 576$ & +16 \\
6 & Red-orange & $\mathrm{RO}$ & $565 / 580$ & $390 / 640$ & +60 \\
7 & Red & $\mathrm{R}$ & $580 / 605$ & $390 / 620$ & +15 \\
8 & Carmine & $\mathrm{CA}$ & $580 / 620$ & $390 / 635$ & +15 \\
9 & Crimson & $\mathrm{C}$ & $625 / 645$ & $390 / 674$ & +29 \\
10 & Scarlet & $\mathrm{S}$ & $645 / 680$ & $390 / 698$ & +18 \\
\hline
\end{tabular}


anomaly in the manufacturing process of the Invitrogen fluospheres. However, it is evident from Fig. 7(d) that the size of bead $O 1$ [see Fig. 7(a)] can be attributed to defocus. While a "best focus" FOV is attempted, some beads contained within the selected FOV are located at different depths. This causes beads to appear larger than their true size.

Once a "best focus" FOV is obtained, we record an intensity-valued spectrally encoded 2D measurement with CASSI. Figure 7(a) shows a CASSI baseline measurement where spectrally coded beads reveal CASSI's optical resolution as a function of wavelength. CASSI measurements $(g)$ are then inverted using the algorithm discussed in Section 3. The reconstructed $\alpha^{*}$ data cube is transformed into a 2D spectral feature map of the scene. Figure 7(b) describes a 2D spectral feature map of a ten bead type fluorescence scene generated from an HSV to RGB mapping, $\gamma_{1}^{*}$. Beads in this $2 \mathrm{D}$ spectral feature map are additionally outlined with white circles. Closer inspection of the reconstructed beads in the $2 \mathrm{D}$ bead identification map reveal artifacts in the bead shape. The artifacts or nonuniformity is caused by local processing of the beads for $\alpha^{*}$ data cube processing. Also, Fig. 7(c) represents a color-based 2D spectral feature map, $\gamma_{2}^{*}$. We add white circles to the $2 \mathrm{D}$

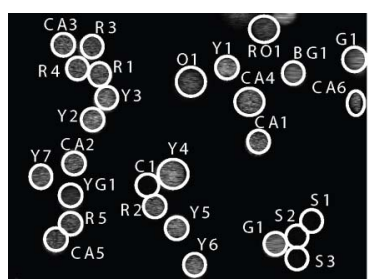

(a)

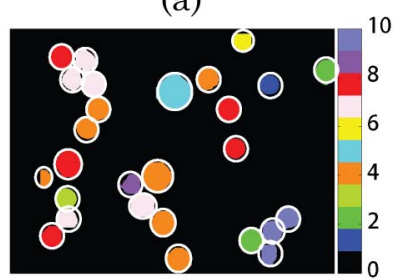

(c)

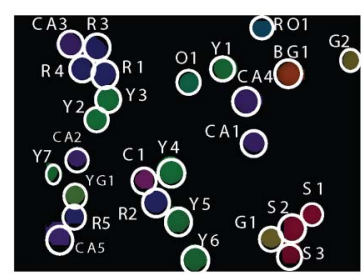

(b)

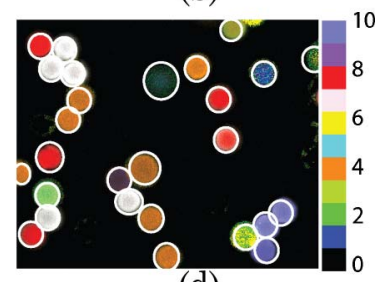

(d)

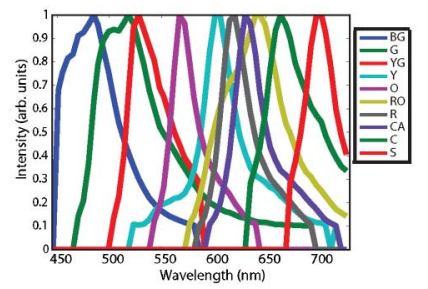

(e)

Fig. 7. (Color online) (a) Baseline CASSI 2D intensity-valued measurement of fluorescent microsphere scenes acquired with a $50 \times, 0.4$ NA microscope objective. White circles are added to the images to outline the locations of the beads. (b) CASSI reconstructed 2D spectral feature map, $\gamma_{1}^{*}$. (c) CASSI 2D spectral feature map, $\gamma_{2}^{*}$. (d) Nikon A1 series baseline image with ten bead type discrimination where beads are additionally outlined in white. (e) Spectral vectors used in the database, $W$, for CASSI reconstructions. bead identification map. Bead reconstruction artifacts from the $\alpha^{*}$ data cube are clearly seen in the color-based 2D map. Again, we chose the TV minimization reconstruction parameter $\tau$ by trial and error and the number of iterations such that the relative difference in the objective function was nominal. Various values of $\tau(\tau=0.5-2)$ are used to evaluate each bead region. Crimson and scarlet beads are weakly excited by UV excitation. Signal intensity from these beads appear dim in the baseline CASSI measurements since well-excited beads provide a higher contrast when compared to the background detector signal. Still, the color-based 2D spectral feature map is able to identify the weakly excited beads despite a loss in the intensity within the bead area due to renormalization of the bead FOV in postdetection.

CASSI measurements and postdetection analysis provide a $2 \mathrm{D}$ spectral feature map where beads are identified based on intensity values and color. In the next discussion, we describe a filtered-array imaging system and present a baseline image that is used to verify spectral feature maps generated from CASSI measurements.

\section{Verification Testbed}

We record a baseline image with a Nikon A1 confocal microscope in order to verify CASSI $2 \mathrm{D}$ bead identification maps. The baseline image is separately recorded and is not associated with the CASSI architecture. We do not compare the result obtained from the baseline system with CASSI reconstructions as it is not fair to do so given different system architectures, variable incident energy at the detector, and different excitation schemes. We show the baseline image only to illustrate ten bead type discrimination with $2 \mathrm{D}$ color coded images produced by a filteredarray system.

We captured a baseline image with a Nikon A1 confocal microscope in a wide-field fluorescence mode. A wide-field fluorescence mode is undertaken since CASSI measurements were recorded in widefield and we were not trying to image diffraction limited spots where out-of-focus light could hinder image analysis. The baseline image was recorded using a CFI Plan-Apochromat 10×, 0.45 NA microscope objective. A multiwavelength illumination scheme was used for ten bead type excitation. A $402 \mathrm{~nm}$ diode laser at $4.8 \mathrm{~mW}$ was used to excite both the blue-green and green beads. Yellow-green and yellow beads were illuminated with a $488 \mathrm{~nm}$ argon line at $8.6 \mathrm{~mW}$. Orange, red-orange, and red beads were illuminated with a $561 \mathrm{~nm}$ laser line at $5.1 \mathrm{~mW}$. The carmine, crimson, and scarlet beads were excited with $640 \mathrm{~nm}$ light at $4.1 \mathrm{~mW}$. The Nikon A1 series is also a grating-based system where dispersed light is focused onto a 32-channel photomultiplier (PMT) array detector so that each channel detects a $10 \mathrm{~nm}$ bandwidth. Since a lambda stack could not be generated at the time of image acquisition, spectra were estimated for various regions of interest, and ten 
spectral emission windows were created in order to isolate each spectrally unique bead type. We recorded a baseline image of $512 \times 512$ pixels (12-bit dynamic range). For the baseline image, colors were manually assigned to each spectrally unique bead type. The bead type colors within Fig. 7(d) were assigned to match the color assignment of the CASSIbased bead identification map in Fig. 7(c). The Nikon A1 series baseline image provided a means for verifying the ten bead type spectral map generated from CASSI measurements.

In Section 5, we described a CASSI-microscope interface that can be used for fluorescence microscopy. In particular, we analyzed a ten bead type fluorescence scene via an adapted TV minimization scheme and a three-step procedure provided a direct method for bead type identification. The Nikon A1 series provided an image that verified CASSI reconstructed spectral maps.

\section{Conclusion}

In this paper, we described a CASSI optical architecture and interface to an upright microscope for fluorescence microscopy. For direct bead identification, we proposed an adaptation of a TV minimization algorithm - the addition of spectral priors in the inversion scheme. This adaptation naturally produced a sparse data cube estimate of a fluorescent scene instead of reconstructing a $f^{*}$ data cube estimate where spectra need to be estimated at every spatial location. With a sparse data cube estimate, we implemented a three-step procedure to produce two spectral identification maps. These spectral identification maps can assist users in localizing fluorescent beads in a $2 \mathrm{D}$ scene. Lastly, a multispectral imaging system, a Nikon A1 confocal microscope, was used to verify CASSI based bead identification maps. Analytical software for the baseline system generated a color-coded image. The Nikon A1 microscope provided a ten bead type spectral identification map that allowed us to verify CASSI spectral maps. Thus, we have provided a direct means for identifying fluorescent beads from CASSI measurements.

We have shown a direct application of CASSI to fluorescence microscopy. Fluorescent beads analyzed in this paper were photon abundant. We were able to use long exposure times to maximize our system SNR. Effects with detector readout noise, background noise, and dark current noise effects more common in low-light fluorescence microscopy would impact TV minimization reconstruction. These effects were not explored in this paper since our system operated under the shot-noise limit. Also, while CS inversion schemes incur a higher computational cost, we have tried to reduce the cost. Since our spectrally encoded measurements are well registered at the detector plane, we were able to apply local processing to the data. Our postdetection process for bead identification was parallizable, and we were thus able to decrease the computational cost. Lastly, we have shown that sparse synthetic data cube estimation in- stead of 3D spectral data cube estimation could potentially reduce an intrinsic computational cost.

We gratefully acknowledge support from the U.S. Air Force Office of Scientific Research (AFOSR), grant 3136057. C. F. Cull acknowledges the support of a National Defense Science and Engineering Graduate Fellowship (NDSEG). The authors would like to thank Bob Guenther for his guidance and support and Nathan Hagan and Andrew Portnoy for their valuable conversations.

\section{References}

1. J. Hackwell, D. W. Warren, R. P. Bongiovi, S. J. Hansel, T. Hayhurst, D. Mabry, M. Sivjee, and J. Skinner, "LWIR/ MWIR imaging hyperspectral sensor for airborne and groundbased remote sensing," Proc. SPIE 2819, 102-107 (1996).

2. A. Goetz, G. Vane, J. Solomon, and B. Rock, "Imaging spectrometry for earth remote sensing," Science 228, 1147-1153 (1985).

3. C. Stellman, F. Olchowski, and J. Michalowicz, "WAR HORSE (wide-area reconnaissance: hyperspectral over-head real-time surveillance experiment," Proc. SPIE 4379, 339-346 (2001).

4. F. Woolfe, M. Maggioni, G. Davis, F. Warner, R. Coifman, and S. Zucker, "Hyperspectral microscopic analysis between normal, benign and carcinoma microarray tissue sections," Proc. SPIE 6091, 60910I (2006).

5. M. L. Huebschman, R. A. Schultz, and H. R. Garner, "Characteristics and capabilities of the hyperspectral imaging microscope," IEEE Eng. Med. Biol. Mag. 21, 104-117 (2002).

6. W. Vermaas, J. Timlin, H. Jones, M. Sinclair, L. Nieman, S. Hamad, D. Melgaard, and D. Haaland, "In vivo hyperspectral confocal fluorescence imaging to determine pigment localization and distribution in cyanobacterial cells," Proc. Natl. Acad. Sci. USA 105, 4050-4055 (2008).

7. M. Descour and E. Dereniak, "Computed-tomography imaging spectrometer: experimental calibration and reconstruction results," Appl. Opt. 34, 4817-4826 (1995).

8. P. Mouroulis, R. Green, and T. Chrien, "Design of pushbroom imaging spectrometers for optimum recovery of spectroscopic and spatial information," Appl. Opt. 39, 2210-2220 (2000).

9. L. Li, X. Qi, X. Hai, and Z. Fa, "Study on microscope hyperspectral medical imaging method for biomedical quantitative analysis," Chin. Sci. Bull. 53, 1431-1434 (2008).

10. S. Gebhart, R. Thompson, and A. Mahadevan-Jansen, "Liquid-crystal tunable filter spectral imaging for brain tumor demarcation," Appl. Opt. 46, 1896-1910 (2007).

11. T. Vo-Dinh, B. Cullum, and P. Kasili, "Development of a multispectral imaging system for medical applications," J. Phys. D 36, 1663-1668 (2003).

12. A. Harvey, D. Fletcher-Holmes, A. Gorman, K. Altenbach, J. Arlt, and N. Read, "Spectral imaging in a snapshot," Proc. SPIE 5694, 110-119 (2005).

13. W. Johnson, D. W. W. Fink, M. Humayun, and G. Bearman, "Snapshot hyperspectral imaging in ophthalmology," J. Biomed. Opt. 12, 014036 (2007).

14. L. Gao, R. T. Kester, and T. Tkaczyk, "Compact image slicing spectrometer (iss) for hyperspectral fluorescence microscopy," Opt. Express 17, 12293-12308 (2009).

15. M. Gehm, S. McCain, N. Pitsianis, D. Brady, P. Potuluri, and M. Sullivan, "Static two-dimensional aperture coding for multimodal, multiplex spectroscopy," Appl. Opt. 45, 2965-2974 (2006).

16. M. Gehm, R. John, D. Brady, R. Willet, and T. Schultz, "Singleshot compressive spectral imaging with a dual-disperser architecture," Opt. Express 15, 14013-14027 (2007). 
17. A. Wagadarikar, N. Pitsianis, X. Sun, and D. Brady, "Video rate spectral imaging using a coded aperture snapshot spectral imager," Opt. Express 17, 6368-6388 (2009).

18. C. Fernandez, A. Wagadarikar, D. Brady, S. McCain, and T. Oliver, "Fluorescence microscopy with a coded aperture snapshot spectral imager," Proc. SPIE 7184, $71840 Z$ (2009).

19. R. Baraniuk, "Compressive sensing," IEEE Signal Process. Mag. 24(7), 118-121 (2007).
20. E. Candes, J. Romberg, and T. Tao, "Robust uncertainty principles: exact signal reconstruction from highly incomplete frequency information," IEEE Trans. Inf. Theory 52, 489-509 (2006).

21. J. Bioucas-Dias and M. Figueiredo, "A new TwIST: two-step iterative shrinkage/thresholding algorithms for image restoration," IEEE Trans. Image Process. 16, 2992-3004 (2007). 